\title{
Efectos de la chicha morada y café sobre el esmalte dental bovino blanqueado con peróxido de hidrógeno.
}

\author{
Effects of chicha and coffee on bovine enamel bleached with hydrogen peroxide.
}

Gisella Castillo-Ghiotto ${ }^{1, a}$, Leyla Delgado-Cotrina ${ }^{1, b}$, Alexis Evangelista-Alva ${ }^{1, c}$.

\section{RESUMEN}

Objetivo: El propósito de este estudio in vitro fue evaluar la susceptibilidad del esmalte dental bovino expuesto a chicha morada y café después del blanqueamiento con peróxido de hidrógeno ( $\mathrm{PH}$ ) al $35 \%$ con y sin calcio. Material y métodos: Setenta y dos dientes de bovino fueron blanqueados con peróxido de hidrógeno al $35 \%$ con y sin calcio (Whiteness HP Blue ${ }^{\circledR}$ - FGM y Whiteness HP Maxx ${ }^{\circledR}$ - FGM, respectivamente) y se expusieron al café instantáneo, refresco de maíz morado artificial y saliva (control) por 30 minutos diarios durante 28 días. El color se midió con el espectrofotómetro Easy-Shade (VITA). El cambio de color se registró a las 24 horas, 7, 14 y 28 días después del blanqueamiento. Las diferencias de color fueron analizados por la prueba de Kruskal Wallis y U de Mann-Whitney.Resultados: Los dientes blanqueados sin calcio expuestos al café fueron estadísticamente más susceptibles a la pigmentación comparado con la chicha morada $(\mathrm{p}<0,05)$. Cuando se utilizó PH al 35\% con calcio no se encontró diferencias estadísticamente significativas $(\mathrm{p}>0.05)$. Conclusión: Los dientes expuestos al café son más susceptibles a la pigmentación que la chicha morada. Los dientes blanqueados con peróxido de hidrógeno al 35\% sin calcio presentan mayor susceptibilidad a la pigmentación por café.

Palabras clave: PERÓXIDO DE HIDRÓGENO, BLANQUEAMIENTO DENTAL, PIGMENTACIÓN, CALCIO.

\section{ABSTRACT}

Objective: The purpose of this study in vitro was to evaluate the staining susceptibility of bovine enamel exposed to chich a and coffee after bleaching. Material and methods: Seventy-two bovine teeth were bleached with 35\% hydrogen peroxide (HP) with and without calcium (Whiteness HP Blue ${ }^{\circledR}$ - FGM and Whiteness HP Maxx ${ }^{\circledR}$ $F G M$, respectively) and were exposed to instant coffee, artificialpurple corndrink and saliva (control) 30 minutes

\footnotetext{
Facultad de Estomatología, Universidad Peruana Cayetano Heredia. Lima, Perú.

a Magíster en Estomatología.

b Docente en Odontología Restauradora y Biomateriales.

c Docente del Departamento Académico de Odontología Social.
} 
daily for 28 days. The color was measured with Spectrophotometer Easy-Shade (VITA). The color change was recorded at 0, 24 hours, 7, 14 and 28 days after bleaching. The color differences were analyzed by the Kruskal Wallis and Mann-Whitney test. Results: The calcium-free bleached teeth exposed to coffee were statistically more susceptible to pigmentation compared with chicha $(p<0.05)$. When using $35 \%$ HP with calcium did not find statistically significant differences ( $p>0.05$ ). Conclusion: Teeth exposed to coffee are more likely to stain than chicha. The teeth bleached with $35 \%$ hydrogen peroxide without calcium show higher susceptibility to pigmentation by coffee.

\section{Keywords: HYDROGEN PEROXIDE, TOOTH BLEACHING, STAINING, CALCIUM.}

\section{INTRODUCCIÓN}

El blanqueamiento vital con peróxido de hidrógeno es una solución estética conservadora en comparación con los tratamientos restauradores que requieren el desgaste previo del tejido dentario. Sin embargo, estudios han reportado que el peróxido de hidrógeno promueve una gran variedad de cambios en el esmalte y dentina como porosidad superficial, cambios en la estructura y permeabilidad, disminución de la microdureza, entre otros $(1,2,3,4,5)$. Por otro lado, se ha demostrado una disminución del contenido de calcio, fosfato y fluoruro del esmalte después de la aplicación del material $(3,6)$. Por tal motivo, se incorporó calcio y flúor en la composición de algunos agentes blanqueadores, con la finalidad de disminuir la pérdida mineral durante el blanqueamiento. Las alteraciones ocurridas por el proceso oxidativo y desmineralización después de la aplicación de los agentes de blanqueamiento podrían contribuir a una mayor pigmentación de la superficie del esmalte cuando es expuesto a ciertas bebidas como el café, gaseosas oscuras, té, jugos naturales y refrescos artificiales. Arévalo y Larrucea afirmaron que los dientes blanqueados presentan mayor cambio de color en el tiempo que los dientes no tratados, al ser sometidos en una solución de café, té y vino (7). Asimismo, Caio y col. encontraron que la desmineralización causada por las sustancias ácidas seran más severa para los dientes blanqueados en comparación con la muestra no blanqueada (8). Algunas soluciones son ácidas y pueden favorecer la desmineralización, mientras que otras pueden presentar pigmentos o colorantes artificiales como la chicha morada que es una bebida muy consumida en la costa del Perú; su insumo principal es el maíz culli, siendo una variedad de maíz morado cultivado en el Perú, Bolivia y Argentina.
Debido a su color morado se presume podría pigmentar la superficie de los dientes. El propósito de este estudio fue evaluar in vitro la susceptibilidad del esmalte dental a la pigmentación frente a la exposición de café y chicha morada después del blanqueamiento con peróxido de hidrógeno al $35 \%$ con y sin calcio.

\section{MATERIALES Y MÉTODOS}

Fueron usados un total de 72 incisivos permanentes de bovino de reciente extracción (Agropecuaria Frigocar SRL, El Camal, Callao, Perú) con un promedio de edad de 3 años. Después de la extracción los dientes fueron limpiados y almacenados en una solución de cloruro de sodio al $0,9 \%$.

Los dientes se distribuyeron aleatoriamente en nueve grupos $(\mathrm{n}=8)$, los cuales fueron distribuídos en Grupos 1a, 1b y 1c: especímenes blanqueados con peróxido de hidrógeno al 35\% (FGM, Whiteness HP Maxx, Joinville, SC, Brasil); Grupos 2a, 2b y 2c: especímenes blanqueados con peróxido de hidrógeno al 35\% con calcio (FGM, Whiteness HP Blue, Joinville, SC, Brasil); Grupos 3a, 3b y 3c: especímenes que no se sometieron al blanqueamiento dental (Control).

Se realizó una profilaxis de las superficies vestibulares con una copa de caucho y una mezcla de piedra pómez y agua. El gel clareador fue aplicado en la superficie vestibular de los dientes con un espesor de película de 0,5 a $1 \mathrm{~mm}$ siguiendo las instrucciones del fabricante. Después del tratamiento se removió el gel con gasa y se lavaron los dientes con agua.

Procedimiento de pigmentación

Las coronas de los especímenes se sumergieron en la solución de café y chicha morada por 30 minutos 
diarios durante 28 días. La solución de café fue preparada a partir de $1 \mathrm{~g}$ de café instantáneo Mónaco (Gloria S.A. Santa Catarina, Lima 13, Perú) en $250 \mathrm{~mL}$ de agua caliente. La chicha morada artificial Negrita (Alicorp SA, Callao, Perú) se preparó a partir de $1 \mathrm{~g}$ de polvo en $200 \mathrm{~mL}$ de agua fría. Especímenes de los grupos 1a, 2a y 3 a fueron sumergidos en una solución de café instantáneo a $64^{\circ} \mathrm{C}$ (promedio de consumo); especímenes de los grupos $1 \mathrm{~b}, 2 \mathrm{~b}$ y $3 \mathrm{~b}$ fueron sumergidos en una solución de chicha morada artificial a temperatura ambiente y los especímenes de los grupos 1c, 2c y $3 \mathrm{c}$ fueron colocados en saliva artificial (Boticas Fasa, La Victoria, Perú). Todas las soluciones se renovaron diariamente.

\section{Medición del color}

El color de todas los especímenes se midió de forma objetiva con el espectrofotómetro Easyshade ${ }^{\circledR}$ Compact Modelo DEASYCS220 (Vita, BadSäckingen, Alemania), basado en la guía de color Vita 3D Master, a la que se le otorgó un número arábico a cada posibilidad de color obteniéndose 110 posibilidades. La medición se realizó tres veces en el tercio medio de la cara vestibular de cada espécimen, inmediatamente posterior a la primera inmersión, a las 24 horas, a los 7, 14 y 28 días. Previo a la medición los especímenes se lavaron con agua y cepillaron por 10 segundos para remover manchas adheridas superficialmente. Después fueron retornados a la saliva artificial y mantenidos a $37^{\circ} \mathrm{C}$.

Los datos obtenidos de las diferencias del color, se analizaron usando el paquete estadístico SPSS 17 para Windows, utilizando la prueba Kruskal Wallis y U de Mann Whitney para muestras independientes con un nivel de significancia del 95\%.

\section{RESULTADOS}

El promedio de las diferencias del cambio de color del esmalte dental del grupo blanqueado con peróxido de hidrógeno $(\mathrm{PH})$ al $35 \%$ sin calcio se muestra en la tabla 1. A las 24 horas, 7, 14 y 28 días después del blanqueamiento, los especímenes blanqueados con $\mathrm{PH}$ sumergidos en café fueron más susceptibles a la pigmentación que los sumergidos en chicha morada $(\mathrm{p}<0,05)$ (Gráfico 1). Para todos los intervalos evaluados después del blanqueamiento no se encontraron diferencias significativas entre los agentes blanqueadores $(\mathrm{p}>0,05)$.

\section{DISCUSIÓN}

En este estudio experimental in vitro se examinó y comparó la susceptibilidad a la pigmentación por café y chicha morada del esmalte blanqueado con $\mathrm{PH}$ al 35\% con y sin calcio. Especímenes de bovinos fueron usados por presentar una composición química y estructura similar a los dientes humanos $(9,10)$.

Los especímenes se almacenaron en saliva artificial luego del proceso de pigmentación para simular la remineralización y estandarizar las condiciones en el estudio. El período de inmersión en las soluciones pigmentantes durante 30 minutos representa un tiempo mayor al comparado con el periodo más corto ocurrido en las condiciones orales diarias, pero se realizó con el objetivo de exponer la muestra a un escenario más extremo para evidenciar la susceptibilidad a la pigmentación con mayor fiabilidad $(11,12)$.

En nuestro estudio, el grupo aclarado con $\mathrm{PH}$ al

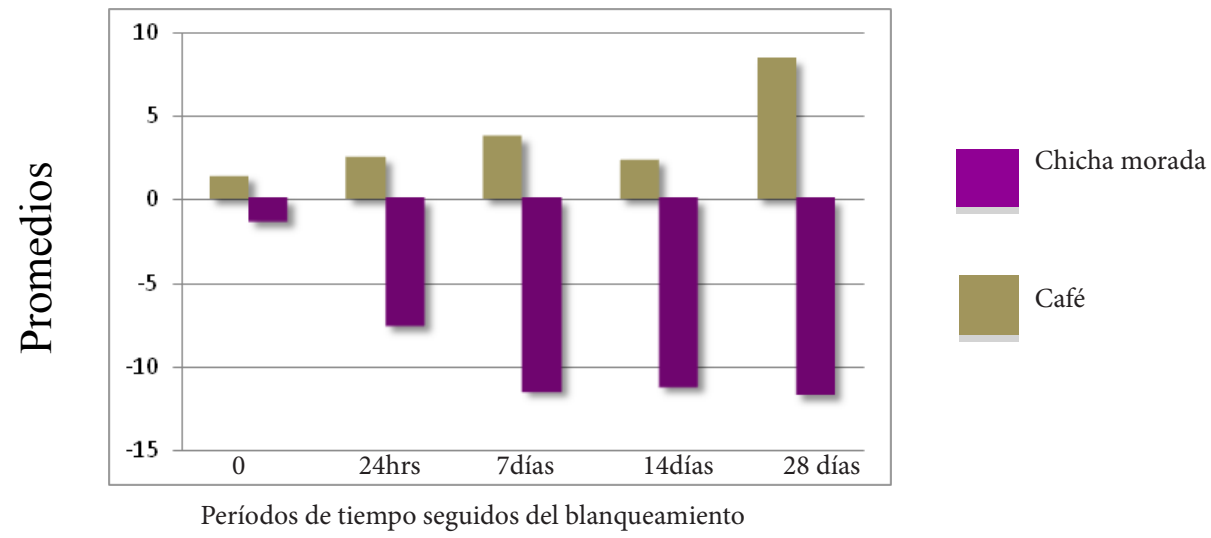

Gráfico 1.Valores promedios de la diferencia de color del esmalte dental bovino blanqueado con peróxido de hidrógeno sin calcio según soluciones pigmentantes. 
$35 \%$ sin calcio, mostró mayor pigmentación por café, comparado con la chicha morada. Al igual que Ghavamnasiri y col. (11), Gross y Col. (13), y Gupta y Col. (14) se encontró que el café es la solución con mayor potencial de pigmentación. Los especímenes sumergidos en chicha morada y saliva artificial fueron más resistentes a la pigmentación.

Esta diferencia se puede deber en parte a la función remineralizante de la saliva que puede revertir los efectos ocasionados por los agentes clareadores sobre la superficie del esmalte como pérdidas de mineral, aumento de la porosidad y rugosidad superficial.

Aunque el café también fue almacenado en saliva artificial, un factor que podría influir a esa tendencia a pigmentar sería la temperatura en la que los especímenes se sumergieron. Estas se mantuvieron a $64^{\circ} \mathrm{C}$ los cuales el promedio de consumo. Pontefract y Col. (15) señalan que a mayor temperatura aumenta la formación de pigmentaciones extrínsecas. Por otra parte, Berger y Col. concluyeron que el tratamiento con peróxido de hidrógeno al 35\% promovió alteraciones en la superficie del esmalte, lo cual incrementó la susceptibilidad a la pigmentación por vino, cuando se comparó con el grupo no blanqueado (16).

Estos cambios en el esmalte y probablemente en la dentina después del blanqueamiento vital pueden conducir a una mayor penetración de bacterias y sustancias colorantes (17).

Entre los especímenes blanqueados con $\mathrm{PH}$ con y sin calcio sumergidas en café y chicha morada no se encontraron diferencias significativas, existiendo una mayor tendencia a la pigmentación por café en la muestra sometida al Whiteness HP Maxx. Esto puede estar asociado a la incorporación del ión calcio en el agente de PH. Giannini y Col. Observaron que las superficies tratadas con agentes blanqueadores que contienen fluoruro y calcio tuvieron similares valores de resistencia a la tracción comparado con las no tra tadas (18). Cavalli y Col. Encontraron que la adición de calcio y flúor en los agentes experimentales (PC 10\%) incrementaron los valores de microdureza del esmalte cuando se comparó con los convencionales (19).

Por otro lado, Borges y Col. Concluyeron que el peróxido de hidrógeno al 35\% reduce significativamente la microdureza superficial del esmalte y que la adición de fluoruro y calcio en el agente blanqueador incrementa los niveles de microdureza del esmalte (20).

Esto puede favorecer a una menor susceptibilidad del esmalte a la pigmentación; pero otro factor que podría asociarse al incremento de la susceptibilidad es la concentración del agente blanqueador, altas concentraciones generan mayor pérdida de calcio $\mathrm{y}$ fos fato del esmalte conduciendo a una superficie denta ria desmineralizada y susceptible a cambiar de color (6).

Los especímenes expuestos a $\mathrm{PH}$ al $35 \%$ sin calcio y expuestos a la solución de chicha morada evidenciaron un aclaramiento progresivo que se podría relacionar a la composición química de la misma. Este hallazgo se podría asociar a un efecto detergente de alguno de los componentes de esta sustancia, la que tendría la propiedad de penetrar y disolver los depósitos de pigmentos sobre las piezas dentarias(21). Los cambios de color más bajos en los valores promedios

Tabla 1.Grado de pigmentación del esmalte dental bovino en relación a períodos y soluciones pigmentantes para Whitness HP Maxx.

\begin{tabular}{lcccc}
\hline \multicolumn{4}{c}{ PERÓXIDO DE HIDRÓGENO al 35\% sin Ca } \\
\hline & \multicolumn{4}{c}{ VALOR $\mathrm{p}^{*}$} \\
Períodos & CROfé & Chichamorada & Saliva artificial & \\
Bl a la 1ra In & $1,38 \pm 1,41$ & $-1,38 \pm 2,20$ & $5,75 \pm 10,38$ & .058 \\
Bl a las 24 hrs & $2,50 \pm 2,98$ & $-7,63 \pm 10,95$ & $-4,13 \pm 4,97$ & .005 \\
B1 a los 7 días & $3,75 \pm 3,81$ & $-11,50 \pm 11,54$ & $-11,50 \pm 10,49$ & .001 \\
B1 a los 14 días & $2,38 \pm 5,97$ & $-11,25 \pm 11,82$ & $-9,00 \pm 10,54$ & .013 \\
B1 a los 28 días & $8,38 \pm 11,75$ & $-11,75 \pm 14,14$ & $-5,38 \pm 9,84$ & .009 \\
\hline
\end{tabular}


fueron registrados para los dientes sumergidos en la solución de chicha morada tanto en los dos grupos experimentales como en el grupo control a los 28 días. Futuros estudios deben realizarse para confirmar estos hallazgos teniendo en consideración el PH de las soluciones que podrían incrementar la desmi neralización (8), o de la presencia de pigmentos que favorecerían a un mayor cambio de color del esmalte.

En conclusión, los especímenes tratados con $\mathrm{PH}$ al $35 \%$ sin calcio son más susceptibles a la pigmenta ción por café en comparación con la chicha morada, y los dientes blanqueados con $\mathrm{PH}$ al 35\% sin calcio presentan mayor susceptibilidad a pigmentarse por café en comparación con el $\mathrm{PH}$ al 35\% con calcio. Los especímenes sumergidos en chicha morada artificial no presentan diferencias en el color dental indepen diente del tratamiento recibido.

\section{CORRESPONDENCIA}

Gisella Castillo Ghiotto

Av. El sol este 137, Lima 04, Perú.

Teléfono: 511-2472870

Correoelectrónico: gisellacg20@hotmail.com

\section{REFERENCIAS BIBLIOGRÁFICAS}

1. Pinheiro H, Costa K, Klautau E, Cardoso P. Análisemicroestructural do esmaltetratado com peróxido de hidrogeno e carbamida. RGO. 2011;59(2):215-20.

2. Cavalli V, Arrais C, Giannini M, Ambrosano G. High concentrated carbamide peroxide bleaching agents effects on enamel surface. J Oral Rehabil.2004;31(2):155-9.

3. Potocnick I, Kosec L, Gaspersic D. Effect of 10 percent carbamide peroxide bleaching gel on enamel microhardness, microstructure, and mineral content. J Endod. 2000;26(4):203-6.

4. Spalding M, Talaveira L, De Asis G. Scanning electron microscopy study of dental enamel surface exposed to $35 \%$ hydrogen peroxide: alone, with saliva, and with $10 \%$ carbamide peroxide. J EsthetRestor Dent. 2003;15(3):154-65.

5. Setien V, Roshan S, Cala C, Ramirez R. Pigmentation susceptibility of teeth after bleaching with 2 systems: an in vitro study. Quintessence Int. 2009;40(1):47-52.

6. Tezel H..Effect of bleaching agents on calcium loss from the enamel surface. Quintessence Int. 2007;38(4):339-47.
7. Arévalo M, Larrucea C. Dental bleaching regression caused by chromogenic beverages. In vitro.RevClinPeriodonciaImplantol.Rehabil Oral. 2012;5(2):57-65.

8. Caio Z. In vitro evaluation of the microhardness of bovine enamel exposed to acid solutions after bleaching. Braz Oral Res. 2011;25(6):562-7.

9. Yassen GH, Platt JA, Hara AT. Bovine teeth as substitute for human teeth in dental research: a review of literature. J Oral Sci. 2011; 53(3):273-82.

10. Schilke R, Lisson J.A, Baub O, Geurtsen W. Comparison of the number and diameter of dentinal tubules in human and bovine dentine by scanning electron microscopic investigation. Arch Oral Biol. 2000;45(5):355-61.

11. Ghavamnasiri M, Bidar M, Rad A, Namazikhah M. The effect of 16 percent carbamide peroxide on enamel staining susceptibility. J Calif Dent Assoc. 2006;34(11):873-6.

12. Attin T, Manolakis A, Buchalla W, Hannig C. Influence of tea on intrinsic colour of previously bleached enamel. J Oral Rehabil. 2003;30(5):488-94.

13. Gross MD, Moser JB. A colorimetric study of coffee and tea staining of four composite resins.J Oral Rehabil. 1977;4(4):311-22.

14. Gupta G, Gupta T. Evaluation of the effect of various beverages and food material on the color stability of provisional materials-An in vitro study. J Conserv Dent. 2011;14(3):287-92.

15. Pontefract H, Courtney M, Smith S, Newcombe RG, Addy M. Development of methods to enhance extrinsic tooth discoloration for comparison of toothpastes. 1.Studies in vitro. J ClinPeriodontol. 2004;31(1):1-6.

16. Berger S, Coelho A, Oliveira V, Cavalli V, Giannini M. Enamel susceptibility to red wine staining after $35 \%$ hydrogen peroxide bleaching. J Appl Oral Sci. 2008;16(3):201-4.

17. Bitter NC. A scanning electron microscope study of the long-term effect of bleaching agents on enamel surface in vivo. Gen Dent. 1998;46(1):84-8.

18. Giannini M, Silva A, Cavalli V, PaesLeme A. Effect of carbamide peroxide based bleaching agents containing fluoride or calcium on tensile strength of human enamel. J Appl Oral Sci. 2006;14(2):82-7.

19. Cavalli V. Effects of bleaching agents containing fluoride and calcium on human enamel. Quintessence Int. 2010;41(8):157-65.

20. Borges AB, Samezima LY, Fonseca LP, Yui KC, Borges $\mathrm{AL}$, Torres $\mathrm{CR}$. Influence of potentially remineralizing agents on bleached enamel microhardness. Oper Dent. 2009;34(5):593-7.

21. Harald M, Eriksen N, Hakon N. Extrinsic discoloration of teeth. J ClinPeriodontol. 1978;5(4):229-36.

Recibido : 16-12-2011

Aceptado: 24-05-2012 